\title{
Experimental Determination of Rigid Body Properties: An Evaluation on the Use of Piezoelectric or MEMS Tri-Axial Accelerometers
}

\author{
António Urgueira, Nuno Venâncio, Pedro Riscado, Raquel Almeida, Tiago Silva* \\ NOVA UNIDEMI, Department of Mechanical and Industrial Engineering, FCT-NOVA, 2829-516 Caparica, Portugal
}

\begin{abstract}
The development of new sensors that are available at more accessible prices may lead to the spread of their use on common studies in structural dynamics. One of areas of interest is the experimental determination of rigid body properties that are mandatory when the vibration response is to be calculated at low frequency ranges. In this work, a comparison of the experimental determination of rigid body properties is carried out to evaluate the performance of the commonly used tri-axial piezoelectric accelerometers and their equivalent MEMS sensors. Although their prices are quite different, both sensors can measure the inertia restraint line that is related to the inertia properties of the tested object. An identification algorithm is applied to the frequency response functions obtained by using both sensors, leading to the estimation of the body mass value, as well as the three coordinates of the centre of mass and the six elements of the inertia tensor. An experimental example supports the use of the referred low-cost sensors.
\end{abstract}

\section{Introduction}

Whenever the dynamic behaviour of a structure at very low frequencies is important, the ten rigid body properties of a mechanical system is of vital importance. These rigid body properties are mass, position of the centre of mass and inertial moments and they may be estimated using numerical or analytical approaches or using experimental driven procedures. The latter have been addressed by several authors [1-10], considering different methods that can be divided into two main categories: Frequency domain methods [1-5] and Time domain methods $[6,7]$.

The Time domain methods [6,7] present advantages mainly due to the fact that there is no need to transform the acquired data into the frequency domain, although care should be taken whenever the structure does not behave like a rigid body in the frequency range of interest. This difficulty can be circumvented by using Frequency domain methods because it is possible to separate the rigid and the elastic system behaviour. The methods based on the Frequency domain are mainly divided into three groups: Inertia Restraint methods (IRM) [1,3], Methods of Direct Physical Parameter Identification [2] and the Modal methods (MM) [4, 1114].

In this work, the authors focused their attention on the use of IRM or the so-called Mass Line methods (MLM) that are based on the principle that the dynamic response of structures in free-free conditions are characterized, in the low frequency region, by a constant term designated as inertial restraint or mass line. This approach is suitable for structures where rigid and flexible modes are well separated. Note that the mass line is the modal constant at zero $\mathrm{Hz}$ of a typical frequency response function (FRF) and can be determined according to the methods described in $[15,16]$. The inertia properties can then be estimated using direct or iterative approaches.

The authors recall here an IRM that was used by several authors [2-4] that is based on several measured accelerations at different locations due to applied excitations at different points. As discussed by Lee [9] the accuracy of this method heavily depends on the selection of the measuring locations, as well as, the conditions of the excitation.

The IRM is here applied to a case study where one has considered the use of two types of sensors, tri-axial piezoelectric accelerometers and their equivalent MEMS sensors, to acquire the measurements at different test configurations. Doing so, one aims at assessing the accuracy of an acquisition system significantly affordable, in economic terms, in comparison to the traditional use of piezoelectric accelerometers. The rigid body properties are experimentally estimated, and the results are discussed.

Note that the MEMS sensor type used is capacitive based displacement accelerometers, which measures the difference of capacitance by the displacement of a proofmass between reference plates [17]. These sensors are lighter and have a very low power consumption when compared to the equivalent piezoelectric accelerometers. The latter have a broader bandwidth, which may be very important for modal studies, but it is not paramount

\footnotetext{
* Corresponding author: tan.silva@fct.unl.pt
} 
regarding the application, where one is only interest in the rigid body motion.

\section{Theoretical background}

As referred, the IRM is based on a set of measured FRFs, specifically accelerance (acceleration/force). In the present work, one uses tri-axial piezoelectric accelerometers in a first stage and after their equivalent MEMS sensors to measure up to three accelerations at a point $p_{i}$ of the structure, in local coordinates. Hence, the vector of measured translational responses at a given location is,

$$
\left\{\ddot{x}_{m_{p_{i}}}\right\}=\left[\begin{array}{lll}
\ddot{u}_{m_{p_{i}}} & \ddot{v}_{m_{p_{i}}} & \ddot{w}_{m_{p_{i}}}
\end{array}\right]^{T}
$$

where $u, v$ and $w$ are the displacements in the three axis, and the double dot represents the second time derivative. Considering $k$ sensors, the vector containing all the responses at different locations can be cast as,

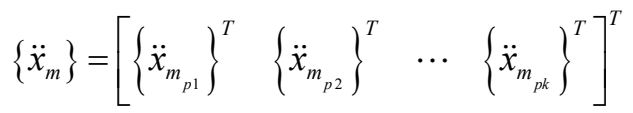

However, one needs to express all the measured responses regarding a global referential for which the rigid body properties are estimated. Thus, using the transformation matrix $\left[G_{m 0}\right]$, one can transform the measured responses to the origin of a given global referential 0 as

$$
\left\{\ddot{x}_{0}\right\}=\left[G_{m 0}\right]^{+}\left\{\ddot{x}_{m}\right\}
$$

where the superscript + is the pseudo-inverse operator. Recalling the Newton's second law, one can relate the exciting forces to the measured responses, being

$$
\left\{f_{0}\right\}=\left[M_{0}\right]\left\{\ddot{x}_{0}\right\}
$$

where $\left[M_{0}\right]$ is a inertia matrix given by

$$
\left[M_{0}\right]=\left[\begin{array}{ccc|ccc}
m & 0 & 0 & 0 & m z_{g} & -m y_{g} \\
0 & m & 0 & -m z_{g} & 0 & m x_{g} \\
0 & 0 & m & m y_{g} & -m x_{g} & 0 \\
\hline 0 & -m z_{g} & m y_{g} & J_{0_{x x}} & -J_{0_{x y}} & -J_{0_{x z}} \\
m z_{g} & 0 & -m x_{g} & -J_{0_{y x}} & J_{0_{y y}} & -J_{0_{y z}} \\
-m y_{g} & m x_{g} & 0 & -J_{0_{z x}} & -J_{0_{z y}} & J_{0_{z z}}
\end{array}\right]
$$

where $m$ is the mass, $x_{g}, y_{g}$ and $z_{g}$ are the coordinates of the centre of mass, and $J_{0_{i j}}$ are the elements of the inertia tensor.

From eq. (4), it is possible to compute all the referred rigid body properties, although one must assure that the number of measurements and exciting forces is sufficient to avoid an ill-posed problem and to fulfil the

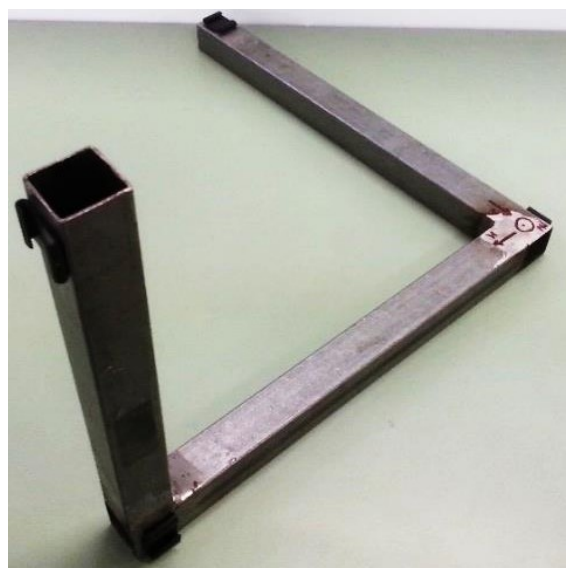

Fig. 1. Rigid body under study.

recommendations of [9]. Note that the measured accelerations of eq. (2) are the mass line constant or the modal constant at zero $\mathrm{Hz}$, that may be obtained using a modal identification or a curve-fitting method.

To demonstrate the applicability of the presented method and to evaluate the use of the MEMS tri-axial accelerometers, in comparison to the traditional piezoelectric ones, one presents the following experimental case study.

\section{Case study}

In this work, one has selected a structure to be studied that has important property (Fig. 1). This structure does not present any plane of symmetry and therefore it exhibits moments and products of inertia when one considers the coordinate system of reference aligned to

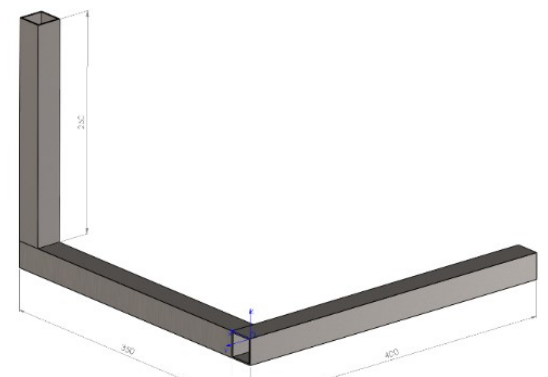

Fig. 2. Model of the rigid body under study (see Fig. 1).

Table 1. Theoretical rigid body properties, regarding the origin of the referential in Figs. 1 and 2.

\begin{tabular}{ccc}
\hline mass $=1.23 \mathrm{~kg}$ & $\rho=7200 \mathrm{kgm}^{-3}$ & \\
$x_{g}=0.169 \mathrm{~m}$ & $y_{g}=-0.089 \mathrm{~m}$ & $z_{g}=0.020 \mathrm{~m}$ \\
$J_{g_{x x}}=0.023029 \mathrm{kgm}^{2}$ & $J_{g_{y y}}=0.023029 \mathrm{kgm}^{2}$ & $J_{g_{z z}}=0.023029 \mathrm{~kg}$ \\
$J_{g_{x y}}=0.023029 \mathrm{kgm}^{2}$ & $J_{g_{x z}}=0.023029 \mathrm{kgm}^{2}$ & $J_{g_{y z}}=0.023029 \mathrm{~kg}$
\end{tabular}




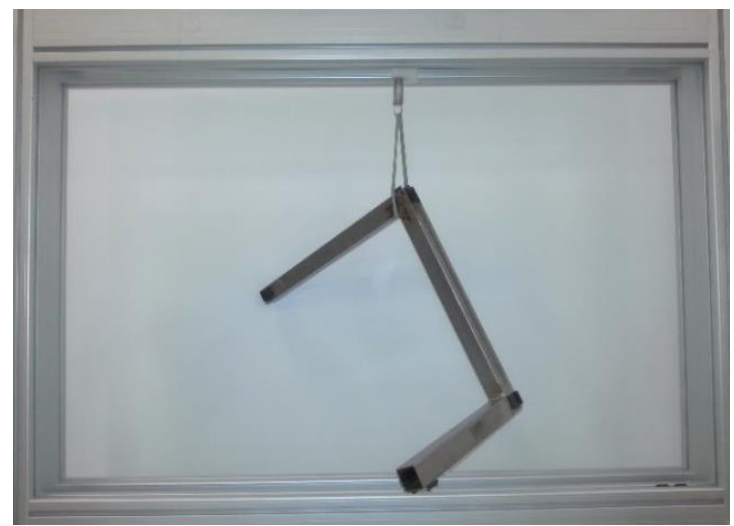

Fig. 3. Soft suspension of the tested structure.

the three components that form the overall structure. These six elements of the inertia tensor together with the three coordinates of the centre of mass and the value of the mass, form the ten unknowns of this experimental method (section 2).

The theoretical model of the structure is built using a CAD software (Fig. 2). The rigid body properties of the model are given in Table 1. These properties will be taken as reference for comparison with the values obtained by the experimental application of the IRM. Note that the reference coordinate system is marked both in Figs. 1 and 2.

The physical structure, shown in Fig. 1 was built up based on the needs of the present experimental work. This structure is made of steel and it was obtained by welding to each other, three rectilinear components.

The experimental work was carried out by assuming the structure to be freely suspended. The soft suspension of the tested structure is shown in Fig. 3, allowing the free movement of the rigid structure, as it is required by the IRM.

The experimental FRFs at different points and directions on the structure were obtained using both type of triaxial translational sensors, the piezoelectrical and

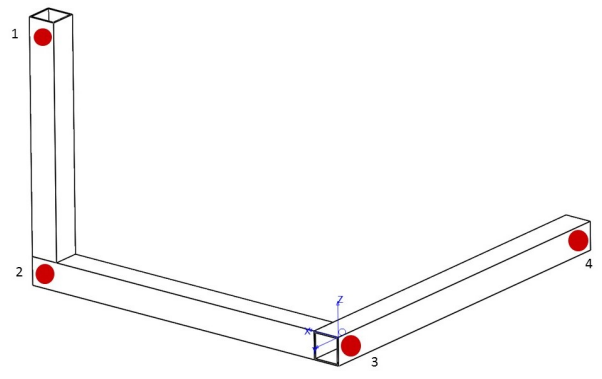

Fig. 5. Reference points on the structure.

Table 2. List of applied forces and sensors positions for each test case (according to Fig. 5).

\begin{tabular}{ccc}
\hline & Measuring configuration & Exciting configuration \\
\hline Test 1 & Conf. A (locations: 1,2,3) & Conf. I (1Y, 2Z, 4X) \\
Test 2 & Conf. B (locations: 1,2,4) & Conf. I \\
Test 3 & Conf. A & Conf. II (1X, 2Z, 2Y) \\
Test 4 & Conf. B & Conf. II \\
\hline
\end{tabular}

MEMS ones, and an instrumented impact hammer. The experimental data was acquired using an acquisition system built at our lab, mainly due to the work of Riscado [18], in the frequency range of $0-400 \mathrm{~Hz}$.

The acquisition system for both types of sensors was built by using a NI DAQ 9172 chassis, as it is shown in Fig. 4. It collects data from the accelerometers triggered by an instrumented impact hammer (PBC 086C03 model). In this work, one has used the tri-axial translational piezoelectric accelerometers B\&K $4506 \mathrm{~B}$ and MEMS sensors ADXL326 (lighter and cheaper).

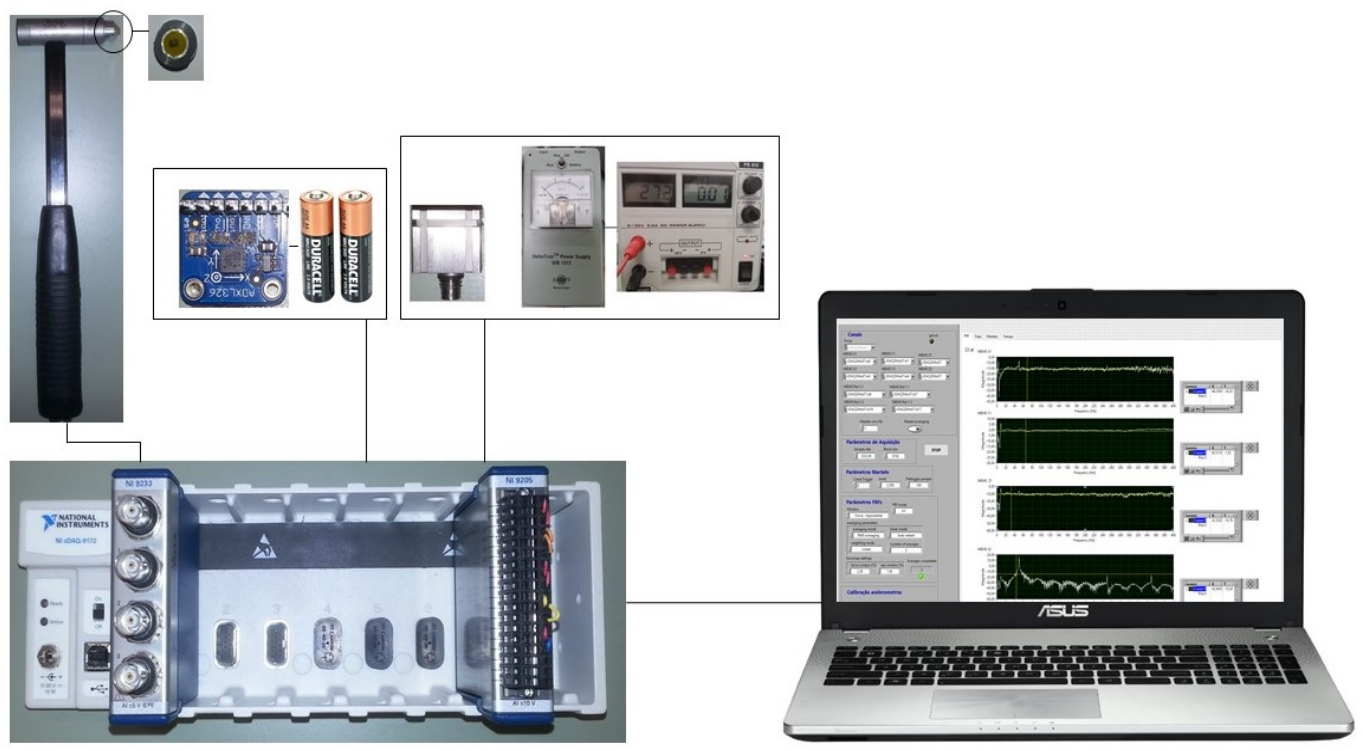

Fig. 4. Schematic representation of the acquisition system used with both types of sensors. 
This acquisition system is implemented using LabView that allows the experimental data to be exported and treated in MatLab, for further developments.

In terms of the experimental procedure, different test cases were considered to evaluate the influence, on the IRM accuracy, of the position and orientation of the sensors and set of applied forces, as described in Table 2 in conjunction with the representation given in Fig. 5.

For each test case (1-4 in Table 2), one measures 9 FRFs per applied force. Note that the exciting configurations given in Table 2 consider a single excitation at a given location and direction, accordingly to a certain axis, and that one uses three different excitations per test.

The entire set of FRFs for each test case is then used of compute the rigid body properties, that cannot be measured directly.

Note that all the pairs sensor/impact hammer were calibrated using a suspended block of known mass.

\section{Results and discussion}

In this section, one shows the most significant results, aiming at evaluating the accuracy of the estimated rigid body properties. Note that the values of the referred properties are shown as a relative error regarding the values of the CAD model (Table 1). Moreover, one also aims at evaluating the performance of the MEMS accelerometers in comparison to the piezoelectric ones.

Hence, from the set of measured FRFs for each test case, here accelerance FRFs (acceleration/force) one obtains the rigid body properties. Figs. 6 and 7 show the values of the relative error for each estimated property, considering the data from both sensors (MEMS in red and piezoelectric in blue). Note that the error at 0 is the one obtained for the comparison of the model value against itself.

From Figs. 6 and 7, it can be inferred that the location of the sensors and of the location and orientation of the exciting forces have a moderated impact on the estimated quantities. Moreover, the use of the MEMS accelerometers is as accurate as the traditional, wellknown, piezoelectrical accelerometers. This is a very important observation, regarding the cost difference between them. From the results, there are no evidences to support a decision towards the choice for a particular type of sensor. It appears that the results accuracy is more related to the configuration of each test case, besides the measurement noise and errors from the identification of the mass line, as there are always some differences in the structure in each test case.
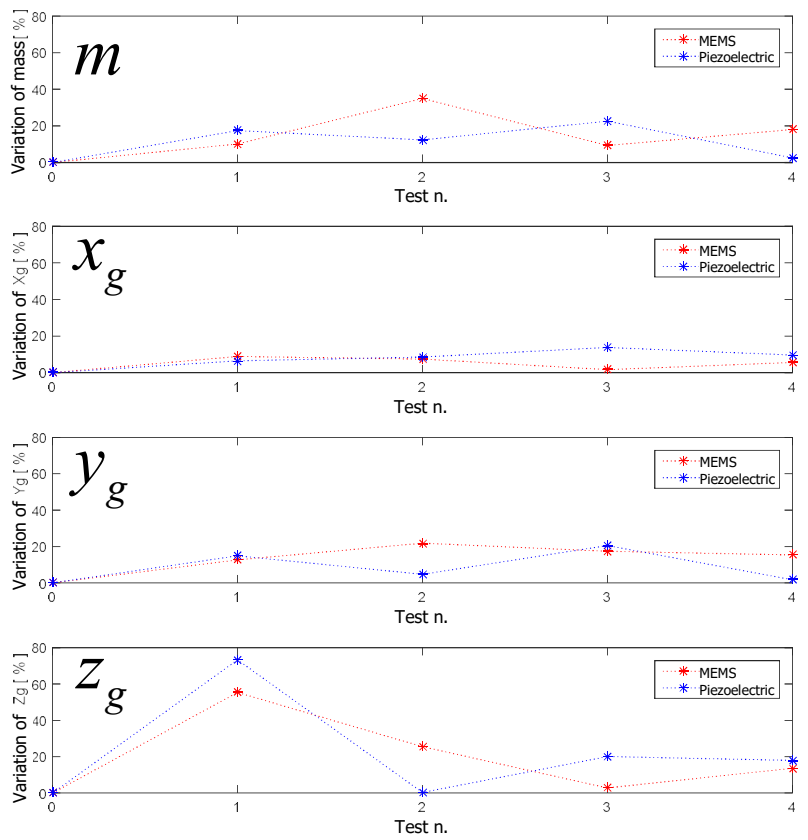

Fig. 6. Relative errors for the estimated rigid body properties for each test case: mass and coordinates of the centre of mass.
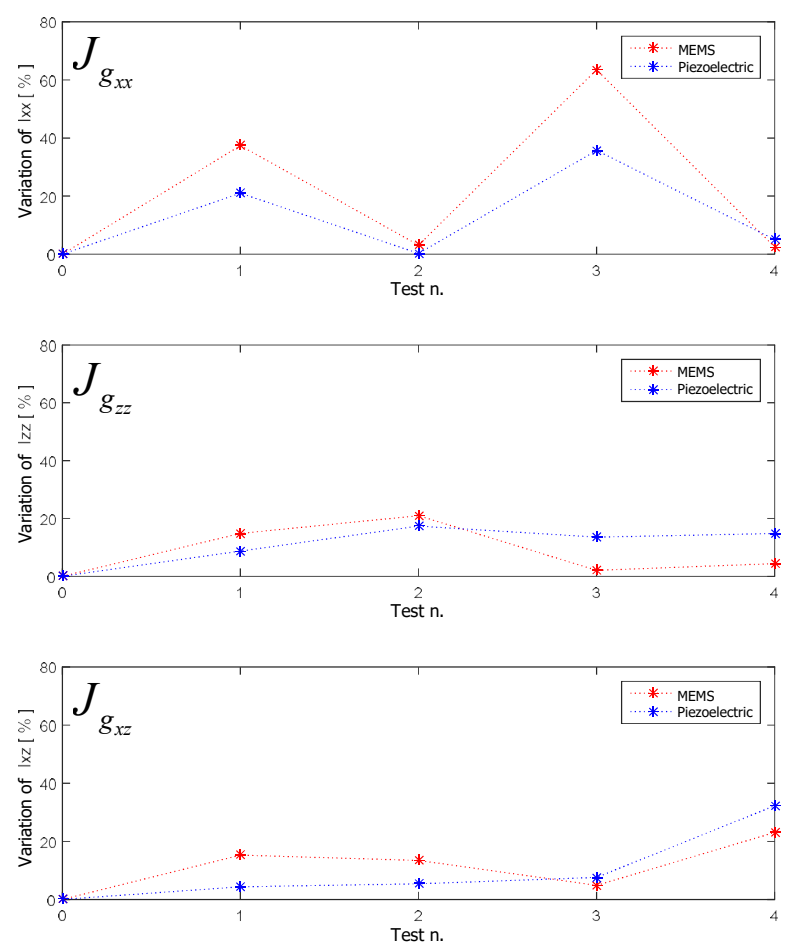

Fig. 7.a. Relative errors for the estimated rigid body properties for each test case: elements of the inertia tensor. 

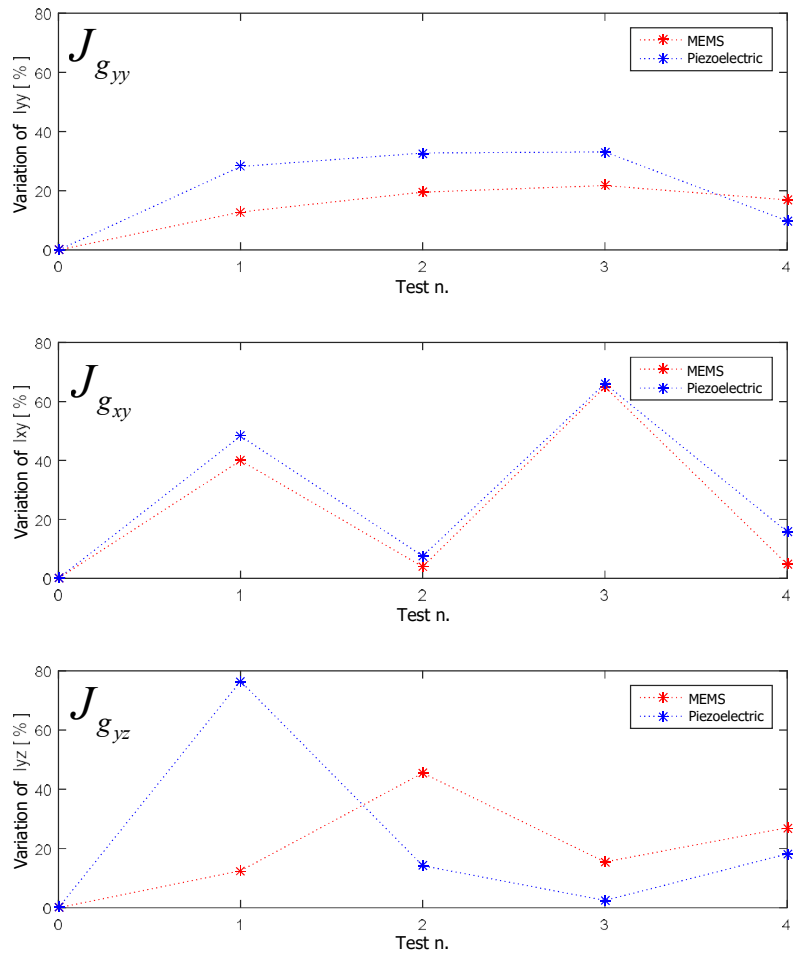

Fig. 7.b. Relative errors for the estimated rigid body properties for each test case: elements of the inertia tensor.

\section{Conclusion}

The IRM is here used to determine the rigid body properties of a non-symmetric structure. The mass line values are extracted from experimental FRFs acquired by two types of tri-axial translational accelerometers, piezoelectric and MEMS. There is no evidence to conclude that the well disseminated piezoelectric sensors have advantage over the MEMS ones. It is important to note that the cost difference between the two types of sensors is very significant. However, as referred in the literature, a special attention to the relative location of the sensors during the testing process is paramount for the accuracy of the estimated rigid body properties, for both types of sensors.

\section{Acknowledgements}

The authors acknowledge the support of the Portuguese Foundation for Science and Technology, FCT, under the strategic project PEst-OE/EME/UI0667/2014 (UNIDEMI, FCT-NOVA)

\section{References}

[1] J. Bretl, P. Conti, in Proc. Int. Modal Analysis Conference IMAC V (1987)

[2] Y. S. Wei, J. Reis, in Proc. Int. Modal Analysis Conference IMAC VII (1989)

[3] A. P. V Urgueira, in Proc. Int. Modal Analysis Conference IMAC XIII (1995)

[4] P. Conti, J. Bretl, J. Vib. Acoust. Stress. Reliab. Des. 111, 2 (1989)

[5] J. Toivola, O. Nuutila, in Proc. Int. Modal Analysis Conference IMAC XI (1993)

[6] S. M. Pandit, Y.-X. Yao, Z.-Q. Hu, J. Vib. Acoust. 116, 3 (1994)

[7] S. M. Pandit, Z.-Q. Hu, J. Sound Vib. 177, 1 (1994)

[8] A. Fregolent, A. Sestieri, Mech. Syst. Signal Pr. 10, 6 (1996)

[9] H. Lee, Y.-B. Lee, Y.-S. Park, Mech. Syst. Signal Pr. 13, 4 (1999)

[10] C. Schedlinski, M. Link, Mech Syst Signal Pr. 15, 1 (2001)

[11] R. A. B. Almeida, A. P. V. Urgueira, N. M. M. Maia, Mech. Syst. Signal Pr. 24, 5(2010)

[12] R. A. B. Almeida, A. P. V. Urgueira, N. M. M. Maia, J. Sound Vib. 299, 4-5 (2007)

[13] R. A. B. Almeida, A. P. V. Urgueira, N. M. M. Maia, Shock Vib. 15, 3-4 (2008)

[14] A. Malekjafarian, M. R. Ashory, M. M. Khatibi, in Proc. Int. Modal Analysis Conference IMAC XXVII (2010)

[15] Silva, Maia, He, Lieven, Lin, Skingle, To, Urgueira, Theoretical and Experimental Modal Analysis, RSP (1997)

[16] D. J. Ewins, Modal Testing: Theory and Practice, RSP (1999)

[17] S. E. Lyshevski, MEMS and NEMS: Systems, Devices, and Structures, CRC Press (2002)

[18] P. Riscado, MSc Thesis, UNL-FCT, Portugal, (2016) 